\title{
Cáncer metastásico con primario desconocido. Una revisión
}

\section{A review of metastatic cancer with unknown primary cancer}

1 Médico, Universidad Nacional de Colombia. Bogotá D. C., Colombia.

2 Profesor de Medicina, Unidad de Gastroenterología, Universidad Nacional de Colombia, Hospital Universitario Nacional de Colombia. Bogotá D. C. Colombia.

3 Internista oncólogo, Instituto Nacional de Cancerología, Universidad El Bosque, Centro Nacional de Oncología. Bogotá D. C., Colombia.

Fecha recibido: 11-09-17

Fecha aceptado: $13-04-18$

\begin{abstract}
Resumen
El tumor metastásico no siempre tiene un origen evidente, hasta en un tercio de los casos nunca se encuentra el tumor primario. Este artículo es una guía de los avances más recientes para mejorar el enfoque diagnóstico y el manejo del paciente con este tumor fatal y frecuente. El objetivo de este artículo, además de ser una guía, es ayudar a evitar errores comunes y graves. Uno de los errores más importantes es no tener en cuenta el papel fundamental de la confirmación histológica, pues esta puede evitar investigaciones innecesarias.

En el artículo también se detallan los componentes de la evaluación estándar, la clasificación según su pronóstico y las indicaciones de la evaluación secundaria, que incluye las indicaciones de la endoscopia alta y baja, los marcadores tumorales, la tomografía por emisión de positrones (TEP), el papel que ocupa el perfil genético, la epigenética y el ácido desoxirribonucleico (ADN) viral. Adicionalmente, se indica el momento en que se debe detener la investigación. Recientemente, el tratamiento se ha modificado, lo que parece cambiar la historia de estos pacientes y de sus contrapartes con primario conocido.
\end{abstract}

\section{Palabras clave}

Tumor metastásico, primario desconocido, cáncer.

\section{Abstract}

Metastatic tumors do not always have obvious origins: in one third of these cases, the primary tumor is never found. This article is a guide to the most recent advances in diagnostic approaches and patient management of these fatal and frequent tumors. An additional objective of this article is to help avoid common and serious errors. One of the most important errors is not taking the fundamental role of histological confirmation into account since it can avoid unnecessary investigations.

The article also details the components of a standard evaluation, classification according to prognosis and indications for a secondary evaluation. These include indications for upper and lower endoscopy, tumor markers, positron emission tomography, and the roles of genetic profiling, epigenetics and viral DNA. It also indicates the moment at which an investigation should be stopped. Recently, treatment has changed, and these changes seems to have changed the history of these patients and their counterparts with known primary tumors.

\section{Keywords}

Metastatic tumor, unknown primary, cancer. 


\section{INTRODUCCIÓN}

El cáncer de primario desconocido (CPD) es un grupo heterogéneo de tumores malignos con confirmación histológica de una de las lesiones metastásicas, en los cuales la lesión primaria no es identificada a pesar de un enfoque diagnóstico estandarizado $(1,2)$. Representa el octavo cáncer más frecuente en el mundo (1), es una neoplasia de mal pronóstico, con supervivencia media de 3 meses (3). En el $10 \%-30 \%$ de los casos, no se logra encontrar el tumor que origina la metástasis aun después realizar una búsqueda exhaustiva con las técnicas más avanzadas -como el perfil molecular- e incluso después de la autopsia (4). Debido a la gran dificultad para encontrar el sitio primario y ofrecer un tratamiento específico, recientemente se han investigado nuevas formas de actuar frente a este tumor en las que se incluyen estudios moleculares, imagenológicos, de inmunohistoquímica y genéticos, que permitan mejorar la supervivencia de estos pacientes $(2,4-7)$.

Teniendo en cuenta la importancia del tema en la práctica clínica diaria y especialmente en gastroenterología, se decidió realizar la siguiente revisión con el propósito de orientar al clínico sobre el enfoque y el manejo de los pacientes con este tipo de presentación oncológica.

\section{METODOLOGíA}

Se establecieron estrategias de búsqueda conformadas por los términos cancer of unknown primary, neoplasms, unknown primary $[\mathrm{MeSH}]$ en asociación con cada punto de interés y se utilizaron términos $\mathrm{MeSH}$ y no $\mathrm{MeSH}$ en español y en inglés. Las estrategias de búsqueda utilizadas fueron: neoplasms, unknown primary AND epigenetic; neoplasms, unknown primary AND immunohistochemical diagnosis; neoplasms unknown primary AND molecular diagnosis; neoplasms, unknown primary AND colonoscopy; neoplasms, unknown primary AND diagnosis; neoplasms, unknown primary AND endoscopy; neoplasms, unknown primary AND treatment. Se realizó la búsqueda de la literatura científica en las bases de datos Pubmed, Embase, Cochrane, Science Direct y Lilacs. Los límites empleados fueron idioma español o inglés, especie humanos y fecha de publicación 2012 a 2017. Se incluyeron aquellos artículos que correspondieran a guías de práctica clínica, estudios observacionales, ensayos clínicos controlados y aleatorizados, y revisiones, revisiones sistemáticas o metaanálisis; de estos, se seleccionaron los que los autores consideraron pertinentes. Adicionalmente, los autores agregaron a la revisión algunos de los artículos mencionados en las referencias de las publicaciones seleccionadas en la búsqueda inicial.

El CPD es un tumor muy agresivo y de mal pronóstico, que a pesar de ser la octava causa de cáncer, es la cuarta causa de muerte por cáncer en el mundo (1, 2, 8). La supervivencia después del diagnóstico varía de 11 semanas a 11 meses (9), con una media de 3 meses (3). Representa el 3\% al 5\% de todos los tumores malignos $(1,2,8)$. Así mismo, tiene una incidencia de 7 a 12 casos/100 000 habitantes/año (2, 10). Cada año en Estados Unidos (EE. UU.) se diagnostican 30000 nuevos casos (9). A medida que aumenta la edad, aumentan los casos con una edad media de presentación de 60-65 años (1). Además de su agresividad, se añade su capacidad metastásica temprana en localizaciones impredecibles $(1,8)$. Esas metástasis afectan 3 o más órganos en un tercio de los pacientes al diagnóstico (8). Los sitios más frecuentes de las metástasis incluyen el abdomen e hígado, seguidos del tórax, cuello y huesos (11). Desde el punto vista histológico, puede clasificarse en 5 subtipos: el subtipo más frecuente es el adenocarcinoma bien y moderadamente diferenciado (60\%), seguido del adenocarcinoma o carcinoma mal diferenciado (29\%), carcinoma escamocelular $(5 \%)$ y las neoplasias malignas mal diferenciadas (5\%) (1).

\section{ENFOQUE INICIAL}

La confirmación histológica y el estudio de inmunohistoquímica del tumor en las metástasis es fundamental y el primer paso para considerar el diagnóstico de CPD, ya que hace parte de la definición del mismo (2). Omitirla sería un grave error que impactaría sustancialmente los desenlaces del paciente, el tiempo de diagnóstico y la realización de exámenes o intervenciones innecesarias, como sucede con mucha frecuencia. No es válida la conducta de ir adelantando antes de la confirmación histológica; es decir, no se justifica realizar endoscopias altas y bajas, y tomografía por emisión de positrones (TEP) mientras se espera el resultado de la biopsia de una metástasis. Igualmente, es necesario realizar la evaluación estándar para poder definir el cáncer de primario desconocido; esta se ha estudiado a profundidad e incluye una historia clínica detallada (1) en la que se evalúan antecedentes familiares y personales de cáncer, síntomas que orientan hacia un probable tumor primario y también factores de riesgo como el tabaquismo $(2,12)$.

El examen físico debe ser completo, se deben buscar masas o adenopatías, y debe incluir la exploración de los senos, piel, pelvis y recto $(1,9)$. No obstante, la importancia de una historia clínica y un examen físico minucioso, de esta enfermedad en particular, con frecuencia no se realiza de esa manera. Si después de lo anterior no se logra saber el origen del tumor primario, se continúa con la siguiente parte de la evaluación estándar, que incluye los siguientes exámenes de laboratorio: hemograma; bioquímica sanguínea (2), que comprende glicemia, electrólitos, calcio, perfil hepático, creatinina, urea y deshidrogenasa láctica (13); uroanálisis; sangre oculta en materia fecal; tomografía axial 
computarizada (TAC) de tórax, abdomen y pelvis $(2,14$, $15)$ con contraste (16), excepto en CPD de cabeza y cuello en nivel nodal 1-3, para el que se sugiere que en la TAC se incluya desde la base del cráneo hasta la pelvis (17).

Aun después de los pasos anteriores reglamentarios, complementados con exámenes más avanzados, en el $20 \%$ a $50 \%$ de los pacientes no se encuentra el tumor que originó la metástasis (18). En estas circunstancias, se estaría realmente ante el diagnóstico verdadero de CPD, siendo esta la definición más estricta $(19,20)$.

Cuando se encuentra el tumor primario en autopsias, los pulmones y el páncreas son los sitios más frecuentes, con un porcentaje de $27 \%$ y $24 \%$, respectivamente $(4,5)$. Otros sitios en los que se han encontrado con frecuencia son el riñón y suprarrenales $(8 \%)$, hígado y vía biliar $(8 \%)$, colon (7\%), sistema genital $(7 \%)$ y estómago $(6 \%)(4,5)$.

\section{FISIOPATOLOGÍA}

Los eventos biológicos que permiten al tumor primario permanecer oculto luego del desarrollo de metástasis aún no se han definido (16). Incluso luego de la autopsia es posible que no se detecte el primario; en estos casos se han planteado teorías, como la de regresión o involución del primario, o el desarrollo de CPD en células madre con capacidad de diferenciación a múltiples líneas celulares (hígado, músculo, piel o incluso células del tracto gastrointestinal), que pueden estar localizadas en el tejido conectivo luego del nacimiento (7). No hay evidencia de que el CPD sea una entidad biológica diferente, con características genéticas o fenotípicas exclusivas en comparación con otros tumores. Diferentes estudios muestran anormalidades cromosomales y aneuploidias, y sobreex- presión de varios genes que no son específicos del tumor de primario desconocido que, por el contrario, se presen$\tan$ en otras malignidades $(10,16,20)$. Las mutaciones y alteraciones genéticas encontradas se han dividido en 6 grupos (Tabla $\mathbf{1}$ ).

\section{CLASIFICACIÓN}

Según su pronóstico, el CPD se divide en 2 grupos: el favorable (que representa el $20 \%$ de los casos) y desfavorable (que representa el 80\% de los casos) (2). La supervivencia promedio de los pacientes en el grupo favorable es de 12 a 36 meses y en el grupo desfavorable es de 6 a 7 meses (19). En el grupo desfavorable, los pacientes usualmente reciben quimioterapia de forma empírica con intención paliativa, pero a pesar de esta tienen un mal pronóstico. Los subgrupos favorables son los más importantes y es a los que van dirigidos todos los esfuerzos, porque al identificarlos, recibirán un tratamiento específico, lo que mejora el pronóstico y algunos pueden tener supervivencia a largo plazo e incluso tener la posibilidad de curación (25-33). Entre ellos se encuentran los siguientes:

- Carcinoma pobremente diferenciado de la línea media de células germinales, que predominantemente afecta a hombres; se presenta en la mayoría de los casos como adenopatía mediastinal o retroperitoneal $(25,34)$.

- Adenocarcinoma seroso papilar peritoneal, que predomina en mujeres y clínicamente se puede presentar como dolor, obstrucción intestinal, masa o ascitis (35).

- Metástasis única, pequeña y potencialmente resecable $(1,2)$.

- Carcinoma escamocelular metastásico de cuello que frecuentemente se manifiesta con adenopatía cervical

Tabla 1. Mutaciones genéticas encontradas en los CPD

\begin{tabular}{|c|c|c|c|c|c|}
\hline Alteraciones cromosómicas & Oncogenes & Supresor tumoral & Vía molecular & Supresor de metástasis & Angiogénesis \\
\hline \multirow{2}{*}{$\begin{array}{l}\text { Aneuploidia } 70 \% \text { de los } \\
\text { pacientes (1) }\end{array}$} & HER2 $(1,2)$ & \multirow[t]{6}{*}{ p53 $(1,8,23)$} & c-Met $(1,24)$ & \multirow{2}{*}{$\begin{array}{l}\text { TIMP-1,(2, 10), MMP2, } 9 \\
(1,2,10)\end{array}$} & $\operatorname{VEGF}(1,2,8,10)$ \\
\hline & EGFR, $(2,21)$ & & pMAPK (25) & & TSP1 (2) \\
\hline \multirow[t]{4}{*}{ Cromosomas 1, 6, 7, 11 (2) } & $C$ kit (1) & & \multirow{2}{*}{$\begin{array}{l}\text { Notch } 3(25) \text {, PTEN } \\
(22,25)\end{array}$} & E-cadherina (25) & CD34 (2) \\
\hline & PDGFR (1) & & & EMT (25) & $\mathrm{HIF}_{10}(25)$ \\
\hline & $\operatorname{BCL2}(2,8)$ & & \multirow{2}{*}{$\begin{array}{l}\text { pAKT, pRPS6 (25), } \\
\text { p21 (25) }\end{array}$} & kiSS1 (2) & \\
\hline & KRAS (22) & & & & \\
\hline
\end{tabular}

BCL2: célula-B CLL/linfoma 2; C kit: receptor de tirosina cinasa; CD34: cúmulo de diferenciación 34; c-Met: proteína del receptor del factor de crecimiento de hepatocitos; EGFR: receptor de factor de crecimiento epidérmico; EMT: transición epitelial a mesenquimal humano; HER2: receptor 2 de factor de crecimiento epidérmico humano; $\mathrm{HIF}_{1 \alpha}$ : subunidad alfa del factor 1 inducible por hipoxia; kiSS1: kisspeptina; KRAS: oncogén viral del sarcoma 2 de la rata Kirsten; MMP: metaloproteinasas; Notch 3: neurogenic locus notch homolog protein 3; p21: inhibidor de la quinasa dependiente de ciclina 1A; p53: proteína supresora tumoral p53; pAKT: proteína cinasa B fosforilada; PDGFR: receptor del factor de crecimiento derivado de plaquetas; pMAPK: proteínas cinasas activadas por mitógenos fosforiladas; pRPS6: proteína ribosomal plastidial S6; PTEN: fosfatidilinositol-3,4,5trisfosfato 3-fosfatasa; TIMP: inhibidor tisular de metaloproteinasas; TSP1: trombospondina 1; VEGF: factor de crecimiento endotelial vascular. Modificado de: Pavlidis N et al. J Adv Res. 2015;6(3):375-82. 
que, en la mayoría de casos, es única y no dolorosa (1); es más frecuente en hombres (80\%) (25).

- Carcinomas neuroendocrinos pobremente diferenciados $(1,2,8)$, que generalmente se localizan en los ganglios linfáticos, hígado o hueso (25).

- Adenocarcinomas que afectan los ganglios axilares en mujeres que tienen comportamiento similar al cáncer de mama $(1,8)$. Después de la mastectomía, se detecta un $70 \%$ de tumores ocultos (25).

- Adenopatía inguinal aislada cuya patología muestra carcinoma escamocelular, en la que se debe buscar el tumor primario en órganos genitales $(1,2)$.

- Hombres con lesiones óseas blásticas con elevación del antígeno prostático cuya patología reporta adenocarcinoma (25).

- Adenocarcinoma con diferenciación de colon, que se puede presentar como metástasis hepática (30\%), adenopatías abdominales $(51 \%)$, metástasis de la superficie peritoneal $(50 \%)$ y ascitis $(27 \%)(25)$.

Por el contrario, la supervivencia en el grupo de pronóstico desfavorable en promedio es de 6 meses a 7 meses incluso con tratamiento (quimioterapia) $(1,6,7,24)$. Los subgrupos de pronóstico desfavorable son:

- Adenocarcinoma metastásico en el hígado u otros órganos.

- Metástasis cerebral múltiple con diferenciación de adenocarcinoma o escamocelular.

- Metástasis pulmonar o pleural múltiple con diferenciación de adenocarcinoma.

- Adenocarcinoma seroso no papilar $(1,2)$.

- Carcinoma pobremente diferenciado.

- Carcinoma escamocelular de la cavidad abdominal (25).

No obstante el mal pronóstico, los investigadores no han perdido el interés y, por el contrario, cada día tienen más entusiasmo y se esfuerzan por investigar y desarrollar múltiples pruebas, entre ellas los estudios endoscópicos, imágenes diagnósticas funcionales, inmunohistoquímica, perfil genético y análisis epigenético $(6,7,36)$.

Cuando el grupo de especialistas tratantes no ha hecho la correcta valoración del paciente o existe la posibilidad de investigaciones adicionales, el diagnóstico en esta etapa intermedia sería CPD provisional (13). Si el paciente no ha sido valorado antes, es en este momento en el que debe ser remitido a oncología. Las indicaciones de los diferentes estudios se describen a continuación.

\section{MARCADORES TUMORALES}

Los marcadores tumorales se han estudiado ampliamente y en la actualidad se considera que tienen baja sensibilidad, especificidad (13) y bajo valor predictivo positivo (VPP) (25). No se consideran diagnósticos, por tanto, no se recomiendan, excepto en las siguientes situaciones:

- Carcinoma de línea media para verificar la diferenciación germinal, en el cual se solicita gonadotropina coriónica humana subunidad $\beta$ (BHCG) (2) y alfa fetoproteína (AFP) (1).

- AFP cuando se sospecha hepatocarcinoma (2), la cual en títulos altos es específica para este tipo de tumor, aunque no se presenta en todos ellos (9).

- Antígeno prostático específico (PSA) en hombres con enfermedad metastásica predominantemente ósea con compromiso blástico $(13,16)$.

El antígeno carbohidrato (CA) 125 y CA 15-3 deben interpretarse con cautela dada su limitada especificidad $(2,13)$.

\section{PROCEDIMIENTOS DIAGNÓSTICOS}

\section{Colonoscopia}

No se realiza rutinariamente ya que no es costo-efectiva (37) y la endoscopia digestiva alta tiene precisión, sensibilidad y especificidad bajas (25). Se recomienda realizar estos 2 exámenes solamente en pacientes con síntomas significativos que sugieran patologías en esos sitios del tracto digestivo y en pacientes con sangre oculta positiva en materia fecal (10) o con hallazgos compatibles por imagen o por histopatología (inmunoperfil sugestivo de adenocarcinoma de colon) (14) (Figuras 1 y 2).

\section{Broncoscopia}

Se realiza en pacientes con factor de transcripción tiroideo 1 positivo, que indica un posible origen pulmonar $(1,15$, 16) y CK 7 positiva (25). En pacientes con adenopatía cervical con histología escamocelular, se debe realizar una panendoscopia, que consiste en laringoscopia indirecta y directa, broncoscopia y endoscopia digestiva alta $(17,38)$.

\section{ESTUDIOS IMAGENOLÓGICOS}

\section{TAC de tórax, abdomen y pelvis}

En ausencia de contraindicaciones, deben realizarse con contraste como estándar en todos los pacientes (16).

\section{Ecografía testicular}

Está indicada en los pacientes con tumor metastásico con diferenciación germinal o en el carcinoma de la línea media $(1,13)$. 


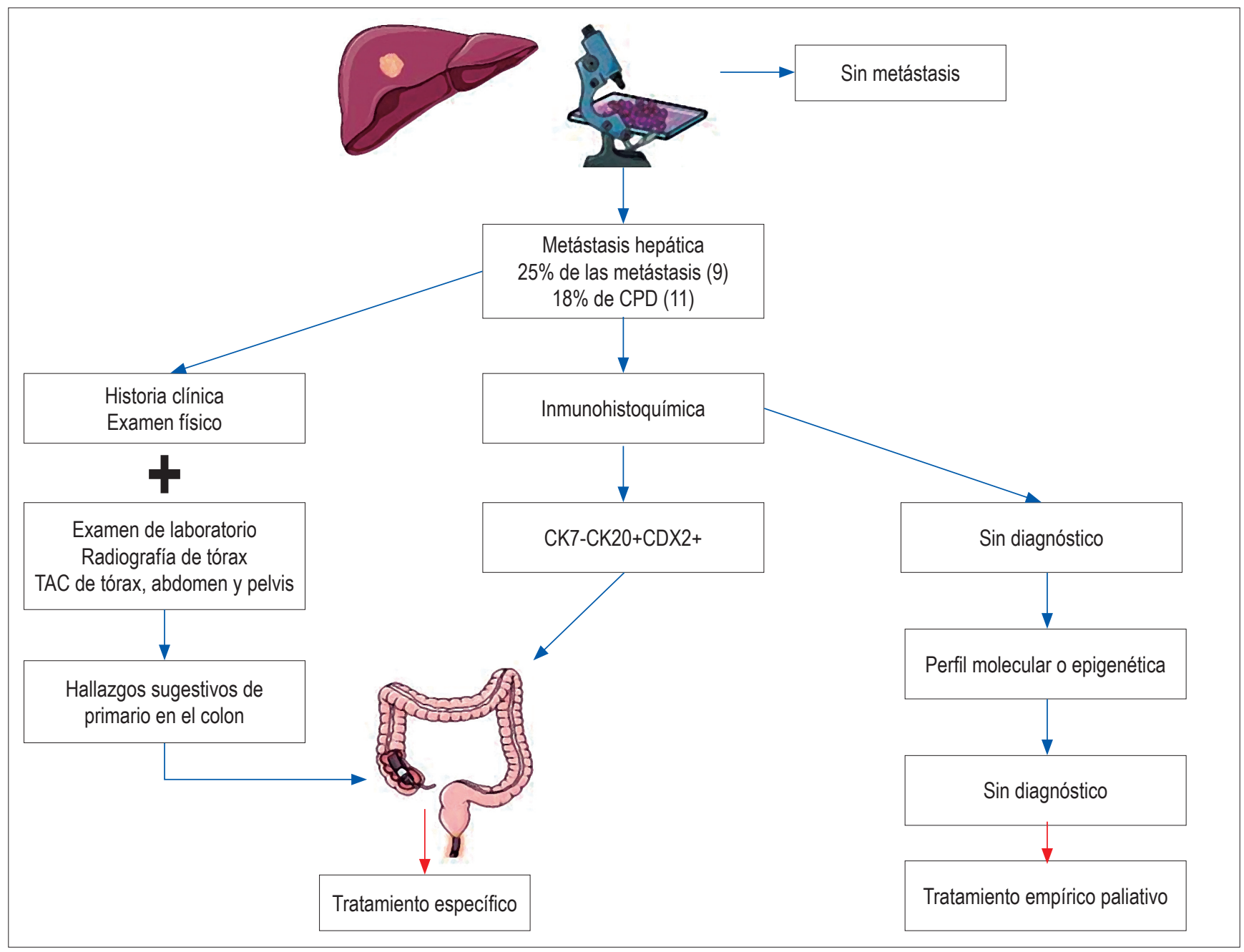

Figura 1. Esquema diagnóstico de metástasis hepática con perfil de adenocarcinoma de colon. CDX2: caudal-related homeobox transcription factor 2; CK: citoqueratina.

\section{Mamografía}

Realizarla de rutina es una conducta errónea (13). Solamente se indica en presencia de síntomas, hallazgos positivos en el examen físico o en la histopatología, y especialmente en pacientes con adenopatía axilar (16).

\section{Resonancia magnética (RM) de mama}

Está indicada en tumores primarios desconocidos con adenopatía axilar, cuando la mamografía es normal. De esta forma, se pueden detectar hasta un $70 \%$ de tumores ocultos (39).

\section{TEP con 5-fluorodesoxiglucosa}

Su uso está actualmente limitado a pacientes con carcinoma escamocelular de primario desconocido metastásico de cuello $(40,41)$. En estos pacientes puede ayudar a guiar la biopsia, determinar la extensión de la enfermedad, facilitar la planeación de la radioterapia y ayudar en el seguimiento de estos pacientes. Se ha encontrado que la TEP puede detectar el primario en un 30\%-45\% de los casos incluso cuando otros estudios imagenológicos no han sido conclusivos, otros estudios favorecen la TEP sobre la panendoscopia en este tipo de pacientes $(40,42)$. Aparte de esta indicación, el rol de la TEP no es claro (16).

\section{TEP con galio}

Otro escenario en que la TEP es útil es el tumor de diferenciación neuroendocrina, en el que la mejor imagen diagnóstica es la PET/TAC DOTA NOC (gallio (68)Ga-labeled $[1,4,7,10$-tetraazacyclododecane-1, 4, 7, 10-tetraacetic acid]$1-\mathrm{NaI}(3)$-octreotide), que es más precisa que el Octreoscan, 


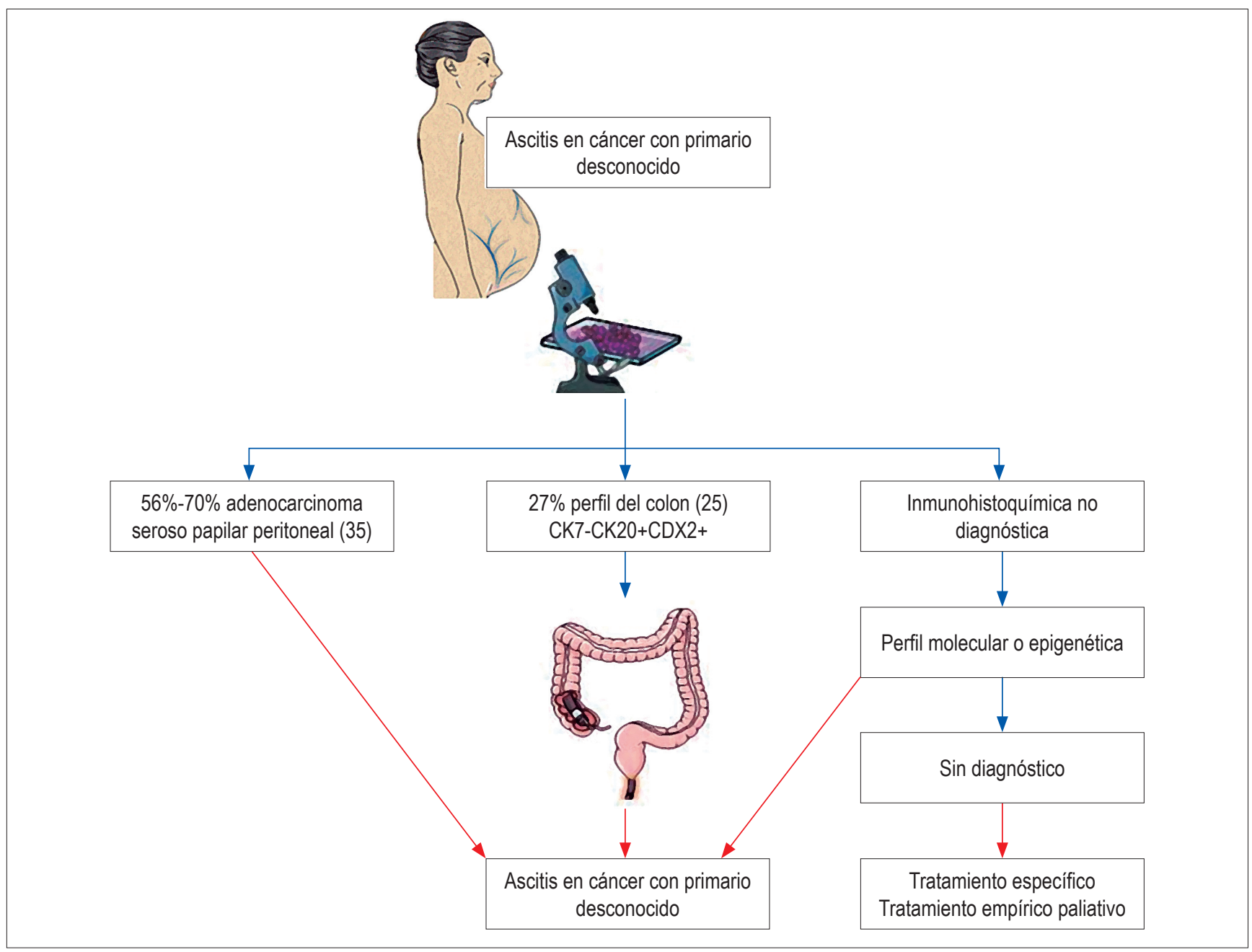

Figura 2. Esquema diagnóstico de ascitis en cáncer con primario desconocido con diferenciación de adenocarcinoma seroso papilar peritoneal o adenocarcinoma con perfil de colon.

TAC y RM (1, 16, 43, 44). El Octreoscan tiene una tasa de detección del $39 \%$ en CPD con diferenciación neuroendocrina (45) y la PET/TAC DOTA NOC en CPD con diferenciación neuroendocrina tiene sensibilidad del 94\%, especificidad del $86 \%$, VPP del $91 \%$, valor predictivo negativo (VPN) del 92\% y precisión del 91\% (43).

\section{INMUNOHISTOQUÍMICA}

La inmunohistoquímica es un procedimiento utilizado por los patólogos, que se basa en la utilización de anticuerpos dirigidos contra las queratinas (familia de proteínas que componen los filamentos intermedios expresadas en carcinomas), factores de transcripción, marcadores de membrana, nucleares y citoplasmáticos, que se usan para definir la diferenciación celular (46).
Es fundamental que el patólogo tenga una adecuada muestra de tejido e información clínica. Con la inmunohistoquímica, el tumor primario se encuentra en el $25 \%$ $30 \%$ de los casos (16); aunque en un metaanálisis reciente se encontró que el rendimiento para detectar el primario puede ser de 65,6\% (47). A pesar de ser el algoritmo más aceptado, se requieren más estudios para establecer si la identificación del tumor primario en grupos que no son de buen pronóstico o ciertos tipos de tumor para los que no hay un tratamiento específico mejora los desenlaces de los pacientes $(1,2,48)$.

Clásicamente, se ha sugerido que el patólogo siga un algoritmo diagnóstico cuando utiliza inmunohistoquímica, y el algoritmo de Pavlidis es el más frecuentemente utilizado (1). Este algoritmo tiene 3 pasos: el primero diferencia linfoma, sarcoma y melanoma, los cuales tienen un manejo 
diferente a los carcinomas; el segundo diferencia los tipos de carcinoma en adenocarcinoma, carcinoma escamocelular, neuroendocrino, tiroideo, renal, hepatocelular y germinal; y el tercero diferencia el tipo de adenocarcinoma, que es muy importante, ya que representa el $80 \%$ de los tumores metastásicos con primario desconocido (49). En el primer paso (Tabla 2) se diferencia el linfoma mediante cúmulo de diferenciación (CD) 45 o antígeno leucocitario común, ya que los linfomas pueden ser positivos para las CK (2); si es positivo para carcinoma, se continúa con el segundo paso $(2,9,25)$.

Tabla 2. Primer paso con inmunohistoquímica

\begin{tabular}{ll}
\hline \multicolumn{1}{c}{ Entidad } & \multicolumn{1}{c}{ Prueba } \\
\hline Linfoma & CD 45 \\
Melanoma & S100, HMB-45 \\
Sarcoma & S100, vimentina \\
Carcinoma & AE1-AE3 pancitoqueratina \\
\hline
\end{tabular}

AE1-AE3: acidic and basic subfamilies of cytokeratin; HMB-45: anticuerpo monoclonal humano melanoma black; S100: multigenic family of nonubiquitous $\mathrm{Ca}^{(2+)}$-modulated proteins. Modificado de: Pavlidis $\mathrm{N}$ et al. Lancet. 2012;379(9824):1428-35.

El segundo y tercer pasos que determinan la diferenciación de carcinomas y adenocarcinomas $(1,25)$ se muestran respectivamente en las Tablas 3 y 4.

Tabla 3. Segundo paso con inmunohistoquímica

\begin{tabular}{ll}
\hline \multicolumn{1}{c}{ Entidad } & \multicolumn{1}{c}{ Prueba } \\
\hline Germinal & Fosfatasa alcalina placentaria, OCT4, AFP, BHCG \\
Neuroendocrina & Cromogranina, sinaptofisina, CD 56, PGP9.5 \\
Renal & RCC, CD 10 \\
Tiroidea & TTF1, tiroglobulina \\
Escamocelular & CK 5 0 CK 6, p63 \\
Adenocarcinoma & CK 7 0 CK 20, PSA \\
Hepática & HepPar1, pCEA canalicular, CD 10, CD 13 \\
\hline
\end{tabular}

HepPar1: anticuerpo hepatocyte paraffin 1; OCT4: octamer-binding transcription factor 4; p63: oncogene belonging to the p53 gene family; pCEA: carcinoembrionario policlonal canalicular; PGP9.5: protein gene product 9.5; RCC: marcador de carcinoma renal; TTF1: factor de transcripción tiroideo 1 . Modificado de: Pavlidis $\mathrm{N}$ et al. Lancet. 2012;379(9824):1428-35.

Otra forma de clasificar los tumores es según las CK 7 y 20, con las que se han creado 4 grupos de tumores (Tabla 5) que pueden sugerir un origen tumoral $(9,50)$. Las CK no son completamente específicas, por lo que no deben ser usadas para invocar un sitio primario en la ausencia de un soporte morfológico o inmunohistoquímico (46).
Tabla 4. Tercer paso con inmunohistoquímica

\begin{tabular}{ll}
\hline \multicolumn{1}{c}{ Entidad } & \multicolumn{1}{c}{ Prueba } \\
\hline Pancreática y biliar & CDX2, CK 20, CK 7 \\
Pulmonar & TTF-1 (2) \\
Colon & CDX-2, CK 20 \\
Prostática & PSA, PAP \\
Mama & Mamaglobina, GCDFP-15, ER (50) \\
Ovario & ER, CA 125, mesotelina, WT1 \\
\hline
\end{tabular}

ER: receptor de estrógenos; GCDFP-15: gross cystic disease fluid protein-15; PAP: fosfatasa acida prostática; TTF-1: factor de transcripción tiroidea 1; WT1: proteína del tumor de Wilms. Modificado de: Pavlidis $\mathrm{N}$ et al. Lancet. 2012;379(9824):1428-35.

Tabla 5. Las CK y tipos de tumores

\begin{tabular}{lll}
\hline \multicolumn{1}{c}{ CK 20 + } & \multicolumn{1}{c}{ CK 20 - } \\
\hline CK 7 + & $\begin{array}{l}\text { Carcinoma urotelial, pancreatobiliar, } \\
\text { mucinoso de ovario, de colon y } \\
\text { broncoalveolar (46) }\end{array}$ & $\begin{array}{l}\text { Pulmonares y } \\
\text { mamarios (46) }\end{array}$ \\
CK 7 - Colorrectal (46) & $\begin{array}{l}\text { Adenocarcinoma } \\
\text { prostático (46) }\end{array}$ \\
\hline
\end{tabular}

Modificado de: Conner JR et al. Adv Anat Pathol. 2015;22(3):149-67.

\section{PERFIL MOLECULAR}

Varios estudios de expresión génica están disponibles en la actualidad; se ha validado su efectividad en identificar el primario en pacientes con primario conocido, con una precisión de un $85 \%$ a $90 \%$. En el caso de pacientes con CPD, se identifica un probable primario en el $70 \%$ a $75 \%$ de los casos (28) mediante tecnología de reacción en cadena de la polimerasa (PCR) en tiempo real de ARN mensajero (mRNA) o micro-ARN (miRNA) o microarrays $(2,9)$. Sin embargo, su impacto y beneficio al dirigir el tratamiento de acuerdo con el resultado del posible primario sigue siendo cuestionable y no probado en ensayos aleatorizados (27, 28). Un estudio prospectivo no aleatorizado fase II de 252 pacientes sugiere que la supervivencia puede mejorarse con estos estudios, en particular para pacientes con tumores sensibles a la quimioterapia al compararse con cohortes históricas. Sin embargo, estos estudios son susceptibles de sesgos, variables de confusión, dada la gran heterogeneidad de primarios desconocidos (16).

Actualmente, se está llevando a cabo en Europa un ensayo clínico fase III para demostrar el beneficio del tratamiento dirigido (por estudio de perfil molecular) frente al tratamiento empírico-NCT01540058 (27).

La posible indicación está dada cuando la inmunohistoquímica y los exámenes de rutina no han logrado establecer un tumor primario $(48,51)$. Aunque no se consideran estu- 
dios que deban realizarse de rutina en todos los pacientes, de acuerdo con las guías internacionales (28).

\section{TRATAMIENTO}

En los subgrupos con pronóstico desfavorable o cuando no se ha podido establecer el tumor primario, el tratamiento de elección es la quimioterapia paliativa basada en platino y taxano (2). Se han estudiado otros esquemas de quimioterapia; sin embargo, en una revisión realizada en el 2000, no se encontró evidencia de superioridad de ningún esquema de quimioterapia en los que se incluyeron sales de platino, taxanos o agentes citotóxicos de nueva generación (gemcitabina, alcaloides de la vinca o irinotecán) $(27,52,53)$. Las tasas de respuesta están alrededor del $20 \%$, con supervivencia media de 6 a 7 meses (1), con o sin quimioterapia $(1,25)$. Sin embargo, en oncología se valoran otros objetivos terapéuticos como la calidad de vida relacionada con la salud, el control de síntomas, los resultados indirectos, la seguridad y los resultados percibidos por los pacientes (54). Una prolongación modesta de la supervivencia y paliación de los síntomas con preservación de la calidad de vida es el objetivo real en estos pacientes, aunque en raros casos se ha reportado curación (27).

Por su parte, los subgrupos favorables reciben principalmente tratamiento regional con cirugía o radioterapia y quimioterapia (1). La supervivencia es similar a la de los tumores metastásicos del mismo origen (48) y el tratamiento también es similar. A continuación, se describe el tratamiento.

\section{Carcinomas pobremente diferenciados de la línea media}

El carcinoma de la línea media recibe quimioterapia con platino, con esquemas similares a los que se utilizan en tumores de células germinales extragonadales; con respuestas completas de un $20 \%$ y parciales de un $25 \%$, y con supervivencia media de 12 meses. Se han reportado tasas de cura de un $10 \%$ a $20 \%(1,8,55)$.

\section{Adenocarcinomas en mujeres con compromiso ganglionar axilar}

Los pacientes con adenopatía axilar se tratan como cáncer de mama y pueden requerir disección ganglionar axilar completa, mastectomía y radioterapia de mamas, quimioterapia adyuvante o terapia hormonal. Cuando esté indicado, es apropiado el uso de trastuzumab (anticuerpos HER2). La supervivencia a los 5 años es de $72 \%$ y a 10 años de $60 \%(1,18)$, aunque la recaída es hasta del $55 \%$ en las pacientes sin terapia local (25).

\section{Carcinomas escamocelulares en ganglios inguinales}

En la adenopatía inguinal se realiza resección y radioterapia con o sin quimioterapia (1). La supervivencia a 5 años se ha estimado de un $20 \%$ (55).

\section{Carcinomas escamocelulares en ganglios cervicales}

Para la adenopatía cervical se realiza disección radical del cuello, con o sin amigdalectomía bilateral y radioterapia (1). En casos seleccionados, se realiza quimioterapia basada en cisplatino concomitante con radioterapia (25). Con estos tratamientos se logra un control locorregional de $80 \%$ a $90 \%$ de los casos, con supervivencia a los 5 años $>65 \%(25)$.

\section{Adenocarcinoma papilar de la cavidad peritoneal}

El adenocarcinoma papilar peritoneal seroso se maneja como un cáncer de ovario estadio III y IV con cirugía y quimioterapia basada en platino y paclitaxel. Un 30\% a 40\% de los pacientes obtiene respuesta completa y un $70 \%$ respuestas parciales, con supervivencia media de 36 meses. Un 16\% de los casos tiene supervivencia a largo término $(7,8,25)$.

\section{Carcinomas neuroendocrinos}

En los tumores con diferenciación neuroendocrina se administra quimioterapia basada en platinos, taxanos, 5-fluorouracilo o capecitabina, temozolomida o irinotecán, con supervivencia media de 15 meses, con respuestas completas hasta de un $21 \%$ (7) y el $13 \%$ de los pacientes presenta supervivencia a largo plazo (18). En el caso de carcinomas neuroendocrinos bien diferenciados, la supervivencia a los 5 años puede ser $>50 \%$ (7).

\section{Adenocarcinomas con inmunoperfil de cáncer de colon}

La metástasis con inmunoperfil de cáncer de colon se tratan como un cáncer de colon metastásico con una supervivencia media de 20 a 36 meses, con tasas de respuestas completas de un $15 \%$ y parciales de un $35 \%$ (25).

\section{Adenocarcinoma con metástasis óseas y elevación de antígeno prostático}

Deben ser manejados como cáncer de próstata metastásico con terapia de deprivación androgénica con o sin quimioterapia basada en docetaxel $(1,7,25)$. La supervivencia de estos pacientes usualmente es $>120$ meses (56). 


\section{CONCLUSIÓN}

El tumor metastásico con primario desconocido causa temor en el paciente y en el médico, pero en esta revisión el médico tiene una guía del enfoque inicial, la posterior clasificación y la indicación de estudios complementarios; además, en ella se destacan los avances científicos recientes que se enfocan en nuevos métodos de diagnóstico y tratamiento dirigidos.

\section{REFERENCIAS}

1. Pavlidis N, Pentheroudakis G. Cancer of unknown primary site. Lancet. 2012;379(9824):1428-35. doi: 10.1016/ S0140-6736(11)61178-1.

2. Natoli C, Ramazzotti V, Nappi O, et al. Unknown primary tumors. Biochimica et biophysica acta. 2011;1816(1):1324. doi: 10.1016/j.bbcan.2011.02.002.

3. Hemminki K, Bevier M, Hemminki A, et al. Survival in cancer of unknown primary site: population-based analysis by site and histology. Ann Oncol. 2012;23(7):1854-63. doi: 10.1093/annonc/mdr536.

4. Pentheroudakis G, Golfinopoulos V, Pavlidis N. Switching benchmarks in cancer of unknown primary: from autopsy to microarray. Eur J Cancer. 2007;43(14):2026-36. doi: 10.1016/j.ejca.2007.06.023.

5. Kim KW, Krajewski KM, Jagannathan JP, et al. Cancer of unknown primary sites: what radiologists need to know and what oncologists want to know. AJR Am J Roentgenol. 2013;200(3):484-92. doi: 10.2214/AJR.12.9363.

6. Moran S, Martinez-Cardus A, Boussios S, et al. Precision medicine based on epigenomics: the paradigm of carcinoma of unknown primary. Nat Rev Clin Oncol.2017;14(11):682694. doi: 10.1038/nrclinonc.2017.97.

7. Tomuleasa C, Zaharie F, Muresan MS, et al. How to diagnose and treat a cancer of unknown primary site. J Gastrointestin Liver Dis. 2017;26(1):69-79. doi: 10.15403/ jgld.2014.1121.261.haz.

8. Pavlidis N, Briasoulis E, Hainsworth J, et al. Diagnostic and therapeutic management of cancer of an unknown primary. Eur J Cancer. 2003;39(14):1990-2005. doi: 10.1016/ S0959-8049(03)00547-1.

9. Swaid F, Downs D, Rosemurgy AS. A practical approach to liver metastasis from unknown primary cancer: What surgeons need to know. Cancer Genet. 2016;209(12):559-566. doi: 10.1016/j.cancergen.2016.08.004.

10. Pavlidis N, Fizazi K. Carcinoma of unknown primary (CUP). Crit Rev Oncol Hematol. 2009;69(3):271-8. doi: 10.1016/j.critrevonc.2008.09.005.

11. Hemminki K, Pavlidis N, Tsilidis KK, et al. Age-dependent metastatic spread and survival: Cancer of unknown primary as a model. Sci Rep. 2016;6:23725. doi: 10.1038/ srep23725.

12. Hemminki K, Chen B, Melander O, et al. Smoking and body mass index as risk factors for subtypes of cancer of unknown primary. Int J Cancer. 2015;136(1):246-7. doi: 10.1002/ ijc.28969.

13. MacReady N. NICE issues guidance on cancer of unknown primary. Lancet Oncol. 2010;11(9):824. doi: 10.1016/ S1470-2045(10)70215-1.

14. Collado Martin R, Garcia Palomo A, de la Cruz Merino L, et al. Clinical guideline SEOM: cancer of unknown primary site. Clin Transl Oncol. 2014;16(12):1091-7. doi: 10.1007/ s12094-014-1244-0.

15. Greco FA. Cancer of unknown primary site: improved patient management with molecular and immunohistochemical diagnosis. Am Soc Clin Oncol Educ Book. 2013:17581. doi: 10.1200/EdBook_AM.2013.33.175.

16. Varadhachary GR, Raber MN. Cancer of unknown primary site. N Engl J Med. 2014;371(8):757-65. doi: 10.1056/ NEJMra 1303917.

17. Muller von der Grun J, Tahtali A, Ghanaati S, et al. Diagnostic and treatment modalities for patients with cervical lymph node metastases of unknown primary site current status and challenges. Radiat Oncol. 2017;12(1):82. doi: 10.1186/s13014-017-0817-9.

18. Massard C, Loriot Y, Fizazi K. Carcinomas of an unknown primary origin--diagnosis and treatment. Nat Rev Clin Oncol. 2011;8(12):701-10. doi: 10.1038/nrclinonc.2011.158.

19. Vajdic CM, Goldstein D. Cancer of unknown primary site. Aust Fam Physician. 2015;44(9):640-3.

20. Kamposioras K, Pentheroudakis G, Pavlidis N. Exploring the biology of cancer of unknown primary: breakthroughs and drawbacks. Eur J Clin Invest. 2013;43(5):491-500. doi: 10.1111/eci.12062.

21. Hainsworth JD, Spigel DR, Thompson DS, et al. Paclitaxel/ carboplatin plus bevacizumab/erlotinib in the first-line treatment of patients with carcinoma of unknown primary site. Oncologist. 2009;14(12):1189-97. doi: 10.1634/ theoncologist.2009-0112.

22. Ross JS, Wang K, Gay L, et al. Comprehensive genomic profiling of carcinoma of unknown primary site: new routes to targeted therapies. JAMA Oncol. 2015;1(1):40-9. doi: 10.1001/jamaoncol.2014.216.

23. Loffler H, Pfarr N, Kriegsmann M, et al. Molecular driver alterations and their clinical relevance in cancer of unknown primary site. Oncotarget. 2016;7(28):44322-9. doi: 10.18632/oncotarget.10035.

24. Stella GM, Senetta R, Cassenti A, et al. Cancers of unknown primary origin: current perspectives and future therapeutic strategies. J Transl Med. 2012;10:12. doi: 10.1186/14795876-10-12.

25. Pavlidis N, Khaled H, Gaafar R. A mini review on cancer of unknown primary site: A clinical puzzle for the oncologists. J Adv Res. 2015;6(3):375-82. doi: 10.1016/j. jare.2014.11.007.

26. Pavlidis N, Petrakis D, Golfinopoulos V, et al. Long-term survivors among patients with cancer of unknown primary. Critical reviews in oncology/hematology. 2012;84(1):8592. doi: 10.1016/j.critrevonc.2012.02.002. 
27. Fizazi K, Greco FA, Pavlidis N, et al. Cancers of unknown primary site: ESMO Clinical Practice Guidelines for diagnosis, treatment and follow-up. Ann Oncol. 2015;26 Suppl 5:v133-8. doi: 10.1093/annonc/mdv305.

28. Ettinger DS, Handorf CR, Agulnik M, et al. Occult primary, version 3.2014. J Natl Compr Canc Netw. 2014;12(7):96974. doi: 10.6004/jnccn.2014.0093.

29. Pentheroudakis G, Lazaridis G, Pavlidis N. Axillary nodal metastases from carcinoma of unknown primary (CUPAx): a systematic review of published evidence. Breast Cancer Res Treat. 2010;119(1):1-11. doi: 10.1007/s10549-009-0554-3.30.

30. Fayanju OM, Jeffe DB, Margenthaler JA. Occult primary breast cancer at a comprehensive cancer center. J Surg Res. 2013;185(2):684-9. doi: 10.1016/j.jss.2013.06.020.

31. Jentsch-Ullrich K, Kalinski T, Roessner A, et al. Longterm remission in a patient with carcinoma of unknown primary site. Chemotherapy. 2006;52(1):12-5. doi: 10.1159/000090235.

32. Lanzer M, Bachna-Rotter S, Graupp M, et al. Unknown primary of the head and neck: A long-term follow-up. J Craniomaxillofac Surg. 2015;43(4):574-9. doi: 10.1016/j. jcms.2015.03.004.

33. Eldeeb H, Hamed RH. Squamous cell carcinoma metastatic to cervical lymph nodes from unknown primary origin: the impact of chemoradiotherapy. Chin J Cancer. 2012;31(10):484-90. doi: 10.5732/cjc.012.10035.

34. Pentheroudakis G, Stoyianni A, Pavlidis N. Cancer of unknown primary patients with midline nodal distribution: midway between poor and favourable prognosis? Cancer Treat Rev. 2011;37(2):120-6. doi: 10.1016/j.ctrv.2010.06.003.

35. Pentheroudakis G, Pavlidis N. Serous papillary peritoneal carcinoma: unknown primary tumour, ovarian cancer counterpart or a distinct entity? A systematic review. Crit Rev Oncol Hematol. 2010;75(1):27-42. doi: 10.1016/j.critrevonc.2009.10.003.

36. Economopoulou P, Pentheroudakis G. Cancer of unknown primary: time to put the pieces of the puzzle together? Lancet Oncol. 2016;17(10):1339-1340. doi: 10.1016/ S1470-2045(16)30377-1.

37. Saliminejad M, Bemanian S, Ho A, et al. The yield and cost of colonoscopy in patients with metastatic cancer of unknown primary. Aliment Pharmacol Ther. 2013;38(6):62833. doi: 10.1111/apt.12429.

38. Mackenzie K, Watson M, Jankowska P, et al. Investigation and management of the unknown primary with metastatic neck disease: United Kingdom National Multidisciplinary Guidelines. J Laryngol Otol. 2016;130(S2):S170-S175. doi: $10.1017 /$ S0022215116000591.

39. Tan S, David J, Lalonde L, et al. Breast magnetic resonance imaging: are those who need it getting it? Curr Oncol. 2017;24(3):e205-e213. doi: 10.3747/co.24.3441.

40. Yoo J, Henderson S, Walker-Dilks C. Evidence-based guideline recommendations on the use of positron emission tomography imaging in head and neck cancer. Clin Oncol (R Coll Radiol). 2013;25(4):e33-66. doi: 10.1016/j. clon.2012.08.007.
41. Rudmik L, Lau HY, Matthews TW, et al. Clinical utility of $\mathrm{PET} / \mathrm{CT}$ in the evaluation of head and neck squamous cell carcinoma with an unknown primary: a prospective clinical trial. Head Neck. 2011;33(7):935-40. doi: 10.1002/ hed.21566.

42. Karapolat I, Kumanlioglu K. Impact of FDG-PET/CT for the detection of unknown primary tumours in patients with cervical lymph node metastases. Mol Imaging Radionucl Ther. 2012;21(2):63-8. doi: 10.4274/Mirt.344.

43. Sharma $\mathrm{P}$, Arora S, Mukherjee A, et al. Predictive value of $68 \mathrm{Ga}-\mathrm{DOTANOC} \mathrm{PET} / \mathrm{CT}$ in patients with suspicion of neuroendocrine tumors: is its routine use justified? Clin Nucl Med. 2014;39(1):37-43. doi: 10.1097/ RLU.0000000000000257.

44. Sadowski SM, Neychev V, Millo C, et al. Prospective Study of 68Ga-DOTATATE Positron Emission Tomography/ Computed Tomography for Detecting Gastro-EnteroPancreatic Neuroendocrine Tumors and Unknown Primary Sites. J Clin Oncol. 2016;34(6):588-96. doi: 10.1200/ JCO.2015.64.0987.

45. Prasad V, Ambrosini V, Hommann M, et al. Detection of unknown primary neuroendocrine tumours (CUP-NET) using (68)Ga-DOTA-NOC receptor PET/CT. Eur J Nucl Med Mol Imaging. 2010;37(1):67-77. doi: 10.1007/ s00259-009-1205-y.

46. Conner JR, Hornick JL. Metastatic carcinoma of unknown primary: diagnostic approach using immunohistochemistry. Adv Anat Pathol. 2015;22(3):149-67. doi: 10.1097/ PAP.0000000000000069.

47. Anderson GG, Weiss LM. Determining tissue of origin for metastatic cancers: meta-analysis and literature review of immunohistochemistry performance. Appl Immunohistochem Mol Morphol. 2010;18(1):3-8. doi: 10.1097/PAI.0b013e3181a75e6d.

48. Greco FA. The impact of molecular testing on treatment of cancer of unknown primary origin. Oncology (Williston Park). 2013;27(8):815-7.

49. Dennis JL, Hvidsten TR, Wit EC, et al. Markers of adenocarcinoma characteristic of the site of origin: development of a diagnostic algorithm. Clin Cancer Res. 2005;11(10):376672. doi: 10.1158/1078-0432.CCR-04-2236.

50. Kandalaft PL, Gown AM. Practical Applications in Immunohistochemistry: Carcinomas of Unknown Primary Site. Arch Pathol Lab Med. 2016;140(6):508-23. doi: 10.5858/arpa.2015-0173-CP.

51. Chiang WM, Kapadia M, Laver NV, Nystrom JS. Cancer of unknown primary: from immunohistochemistry to gene expression profiling. J Clin Oncol. 2012;30(29):e300-2. doi: 10.1200/JCO.2011.41.1827.

52. Golfinopoulos V, Pentheroudakis G, Salanti G, et al. Comparative survival with diverse chemotherapy regimens for cancer of unknown primary site: multiple-treatments meta-analysis. Cancer Treat Rev. 2009;35(7):570-3. doi: 10.1016/j.ctrv.2009.05.005.

53. Amela EY,Lauridant-Philippin G, Cousin S, et al. Management of "unfavourable" carcinoma of unknown primary site: 
synthesis of recent literature. Crit Rev Oncol Hematol. 2012;84(2):213-23. doi: 10.1016/j.critrevonc.2012.03.003.

54. Gaertner J, Weingartner V, Lange S, et al. The role of end-oflife issues in the design and reporting of cancer clinical trials: A structured literature review. PloS one. 2015;10(9):e0136640. doi: 10.1371/journal.pone.0136640.

55. Greco FA. Molecular diagnosis of the tissue of origin in cancer of unknown primary site: useful in patient manage- ment. Curr Treat Options Oncol. 2013;14(4):634-42. doi: 10.1007/s11864-013-0257-1.

56. Takagi T, Katagiri H, Kim Y, et al. Skeletal metastasis of unknown primary origin at the initial visit: a retrospective analysis of 286 cases. PLoS One. 2015;10(6):e0129428. doi: 10.1371/journal.pone.0129428. 